\title{
The Effect of Blue Light Exposure on the Expression of Circadian Genes: Bmall and Cryptochrome 1 in Peripheral Blood Mononuclear Cells of Jaundiced Neonates
}

\author{
AN CHEN, LIZHONG DU, YAPING XU, LIQING CHEN, AND YIDONG WU \\ Neonatal Intensive Care Unit, the Children's Hospital of Zhejiang University School of Medicine, \\ Hangzhou 310003, P R China
}

\begin{abstract}
The purpose of this study was to investigate the effect of blue light phototherapy on the expression of circadian genes in peripheral blood mononuclear cells (PBMC) and plasma melatonin levels in neonates. Real-time reverse-transcriptase polymerase chain reaction (RT-PCR) was used to determine the expression of Bmall and Cryl in PBMC, and an enzyme-linked immunosorbent assay was used to determine plasma melatonin levels in 32 breast-milk jaundiced neonates before and after phototherapy, compared with 29 control neonates. The results showed that the expression of Bmall was decreased and Cryl increased significantly after phototherapy. Plasma melatonin levels were decreased after phototherapy. There was no statistical difference in Bmall and Cryl gene expression and plasma melatonin levels in
\end{abstract}

ABSTRACT

the control group. In conclusion, phototherapy does affect the expression of the circadian genes Bmall and Cryl in PBMC and plasma melatonin concentration in jaundiced neonates. Our results suggest that phototherapy should be timed according to circadian rhythms when treating jaundiced neonates. (Pediatr Res 58: 1180-1184, 2005)
Cry, cryptochrome
PBMC, peripheral blood mononuclear cells
RHT, retinohypothalamic tract
SCN, suprachiasmatic nuclei

Abbreviations
Circadian rhythm refers to an endogenous biologic clock with a period of approximately 24 hours. In mammals, the circadian pacemaker is located in the suprachiasmatic nuclei (SCN) of the anterior hypothalamus. There is some evidence that circadian rhythms develop prenatally (1). At least 12 genes have been reported to be involved in regulating the circadian rhythm (2). These are Clock, NPAS2, Bmal1, Period1, Period2, Period3, REV-ERB ${ }_{\alpha}, C r y 1, C r y 2, D e c 1$, Dec2, and Tau (casein kinase $\mathrm{I} \varepsilon$ ). They constitute transcriptional-translational feedback loops, in which Period1, Period2, Period3, REV-ERB ${ }_{\alpha}$, Cry1, Cry2, Dec1, and Dec2 are negative regulators, whereas Clock, NPAS2, and Bmall are positive regulators. Tau binds and phosphorylates PER proteins, posttranscriptionally regulating their stability. These genes oscillate not only in the SCN, but also at the peripheral tissues, such as skin, oral mucosa, and PBMC $(3,4)$.

Received November 10, 2004; accepted April 29, 2005

Correspondence: Lizhong Du, M.D., Neonatal Intensive Care Unit, the Children's Hospital, Zhejiang University School of Medicine, \#57 Zhu Gan Lane, Hangzhou 310003, P.R. China: e-mail: dulizhong@yahoo.com.cn

DOI: 10.1203/01.pdr.0000183663.98446.05
Melatonin is the main hormone secreted by the pineal gland, which is connected to the SCN by a multisynaptic pathway. The synthesis and release of melatonin are promoted by darkness and inhibited by light. The roles of melatonin in the control of circadian rhythm and human sleep have been extensively investigated by many authors $(5,6)$. The significance of this hormone in the regulation of human circadian behavior is controversial, especially among neonates who have been exposed to phototherapy $(7,8)$. Light-dark cycle change in the environment may be detected by the SCN through the RHT (2), or through nonocular photoreceptors $(9,10)$. Light-dark cycle change can entrain the SCN to synchronize with the environment.

Jaundiced neonates are usually treated with phototherapy and blue light is used as the main light source. Light reduces the serum bilirubin level by converting poorly soluble indirect bilirubin into water-soluble photoisomers that can be excreted into bile and urine without liver conjugation. When neonates are exposed to the blue light during phototherapy, their eyes are covered to avoid retinal damage. One of the most common clinical findings in the behavior of neonates during phototherapy is that they often present with an increased rate of crying 
and jitteriness. Whether this nonocular light exposure could influence the expression of circadian rhythm in these neonates and how it is related to their vital signs or behavior change have not previously been investigated. In our present study, we examined the expression of Bmall and Cryl genes in the PBMC of breast-milk jaundiced neonates before and after phototherapy and tried to elucidate the influence of phototherapy on the expression of circadian genes. We also measured the plasma melatonin levels in these neonates to examine a possible association with the regulation of circadian behavior during phototherapy.

\section{METHODS}

Subjects. Sixty-one jaundiced full-term neonates, aged from 12 to 27 days, were recruited in the neonatal ward of Zhejiang Children's Hospital between September 2003 and May 2004. The study was approved by the hospital review board for the protection of human subjects, and informed consent was obtained from all parents following a detailed explanation of the study.

All infants were hospitalized for hyperbilirubinemia. Infants with birth asphyxia, prematurity, infection, or hemolytic diseases were excluded from the study. The primary diagnosis was an exaggerated physiologic jaundice, such as breast milk jaundice, prolonged physiologic jaundice, or a high level of unconjugated bilirubinemia of unknown origin. Vital signs were monitored throughout the study. The infants were fed breast milk before hospitalization and an infant formula after admission. Routine laboratory examinations were all normal except for high serum bilirubin levels. Total serum bilirubin was $10-15 \mathrm{mg} / \mathrm{dL}$ with $<1.5 \mathrm{mg} / \mathrm{dL}$ of direct bilirubin level, as determined by spectrophotometer. At this borderline serum bilirubin level, the babies can be treated either by phototherapy or simple observation according to the recommended guidelines of the Chinese Neonatal Society (11). We randomly divided all neonates into two groups. Group I, the treatment group, consisted of 32 neonates treated with a double-sided blue light phototherapy device, a light box with twelve 20-W cool fluorescent bulbs (Ningbo David Medical Device Co. Ltd., China) starting on the second day after hospitalization. The phototherapy lasted for 24 hours from $0010 \mathrm{~h}$ to $0010 \mathrm{~h}$ the next day. During the therapy, eyes and perineum were covered with black cloth and the bodies were exposed to the blue light. Group II, the control group, consisted of 29 neonates not treated with phototherapy. However, on the second day of their hospitalization, their eyes were also covered with black cloth for 24 hours from $0010 \mathrm{~h}$ to $0010 \mathrm{~h}$ the next day. During this period, they were clothed. In the neonatal ward, the room lights were not turned off. Measurements of irradiance were made at the level of the infant either within the light box for the treatment group or in the room light environment for control infants by an illuminance meter (Minolta T-10 Illuminance Meter). Total irradiance was 5500-7200 lux $\left(806-1054 \mu \mathrm{W} / \mathrm{cm}^{2}\right)$ in group I and $72-85$ Lux in group II.

Sample acquisition. Within a half hour before and after phototherapy in group I, or before and after the eyes were covered in group II, $1.5 \mathrm{~mL}$ of peripheral venous blood sample was obtained from the neonate and collected in a sterile tube with EDTA- $\mathrm{K}_{3}$ anticoagulant. The blood was processed by centrifugation immediately. Plasma was separated and kept at $-80^{\circ} \mathrm{C}$ for melatonin determination. PBMC were isolated using the Ficoll-Paque density gradient centrifugation method, then washed and frozen at $-80^{\circ} \mathrm{C}$ until the assay.

Total RNA isolation and reverse transcription. Total cellular RNA was isolated from PBMC samples using TRIzol reagent according to the manufacturer's description (GIBCO BRL). RNA concentration was determined by spectrophotometry. RT reactions were carried out for each RNA sample in MicroAmp reaction tubes using RevertAid First Strand cDNA Synthesis Kit (Fermentas) according to the manufacturer's protocol.

Plasmid construction. We used plasmid to construct standard curves. We first designed primers covering two or three introns of human homologues of the circadian genes in the GeneBank. The sequences were hBmall: forward (f) CTCCAGCCCATTAACATC, reverse (r) GCTACCAATGATGCTTCTGT, product size 638 bp. hcryl: f-TCCCAGGTTGTAGCAGCAGT, r-ATTTGGATTACGCACATTAT, 670 bp. $h G A P D H$ : f-GAAGGTGAAGGTCGGAGTC, r-GAAGATGGTGATGGGATTTC, 225 bp. Then we performed PCR in a $50-\mu \mathrm{L}$ reaction mixture containing $2 \mu \mathrm{L}$ of cDNA, $1 \mu \mathrm{L}$ of $0.2 \mu \mathrm{M}$ of each primer, $1 \mu \mathrm{L}$ of $10 \mathrm{mM}$ dNTP, $5 \mu \mathrm{L}$ of $10 \times$ PCR buffer, $4 \mu \mathrm{L}$ of 25 $\mathrm{mM} \mathrm{MgCl} 2$, and $2.5 \mathrm{U}$ of Taq polymerase. Each PCR reaction consisted of one cycle at $94^{\circ} \mathrm{C}$ for 5 minutes, followed by 40 cycles at $94^{\circ} \mathrm{C}$ for 30 seconds, $55^{\circ} \mathrm{C}$ for 30 seconds, and $72^{\circ} \mathrm{C}$ for 30 seconds, with an additional cycle of
$72{ }^{\circ} \mathrm{C}$ for 7 minutes at the end of the reaction. Products were purified, retrieved, and then cloned.

Real-time PCR. We constructed further primers and probes including two introns of the desired genes. The sequences were as follows: hBmall: fCTCCAGCCCATTAACATC, r-GCCTCATCATTACTGGGACT, probe, 5'FAM-CTCCCCCTGATGCCTC TTCTCC-TAMRA-3', product size 240 bp. hcryl: f-GCAGTGGAAGTTGCTC TCAAG, r-CTGAATGTTTTCTAATTAGTGC, probe, 5'FAM-AGGTGAGTTTGC TGACTGTCGCCATTAMR A-3', 196 bp. hGAPDH: f-GAAGGTGAAGGTCG GAGTC, rGAAGATGGTGATGGGATTTC, probe, 5'FAM-CAAGCTTCCCGT TCTCAGCC-TAMRA-3', 225 bp. Then we performed PCR in a $50-\mu \mathrm{L}$ reaction mixture containing $2 \mu \mathrm{L}$ of cDNA, $1 \mu \mathrm{L}$ of $0.2 \mu \mathrm{M}$ of each primer, $1 \mu \mathrm{L}$ of $10 \mathrm{mM}$ dNTP, $5 \mu \mathrm{L}$ of $10 \times$ PCR buffer, $7 \mu \mathrm{L}$ of $25 \mathrm{mM} \mathrm{Mgcl}_{2}, 2.5$ $\mathrm{U}$ of Taq polymerase, and $0.4 \mu \mathrm{L}$ of $0.2 \mu \mathrm{M}$ of each probe. Each PCR reaction consisted of one cycle at $94^{\circ} \mathrm{C}$ for 5 minutes, followed by 40 cycles at $94^{\circ} \mathrm{C}$ for 30 seconds, $55^{\circ} \mathrm{C}$ for 45 seconds, and $72^{\circ} \mathrm{C}$ for 40 seconds using a GeneAmp 5700 Sequence Detection System (Applied Biosystems), according to the manufacturer's instructions. Water controls were included to ensure specificity. Circadian genes mRNA abundance of expressed in molecules was normalized to the abundance of GAPDH mRNA to control for sample-to-sample variation in total RNA input and RT efficiency. The real-time PCR curves are depicted in Figure 1.

Melatonin assay. Plasma melatonin levels were assayed with an ELISA kit (IBL Immuno-biological Laboratories) according to the manufacturer's directions.

Statistical analysis. Patient characteristic values are expressed as mean \pm SD. Qualitative variables were compared with a $\chi^{2}$ test. An independentsamples $t$ test was used for quantitative variables of patient characteristics. Because the variables of circadian gene expression were not a normal distribution, we used nonparametric test to analyze them. A paired nonparametric test (Wilcoxon matched-pair test) for quantitative variables was used for paired observations, i.e. before versus after phototherapy in group I or before versus after 24 hours of eye coverage in control patients. The Mann-Whitney $U$ test was used to compare the baseline circadian genes expression or baseline melatonin levels between groups I and II. We determined the change score for each group by calculating the respective difference between baselines to post-treatment or post-24 hour control of each infant. Statistical significance was defined as $p<0.05$. SPSS 11.5 software was used for statistical analysis and GrathPad Prism 4 was used for graph presentation.

\section{RESULTS}

Patient characteristics. Patient characteristics are summarized in Table 1 . In group I, 17 babies were delivered by
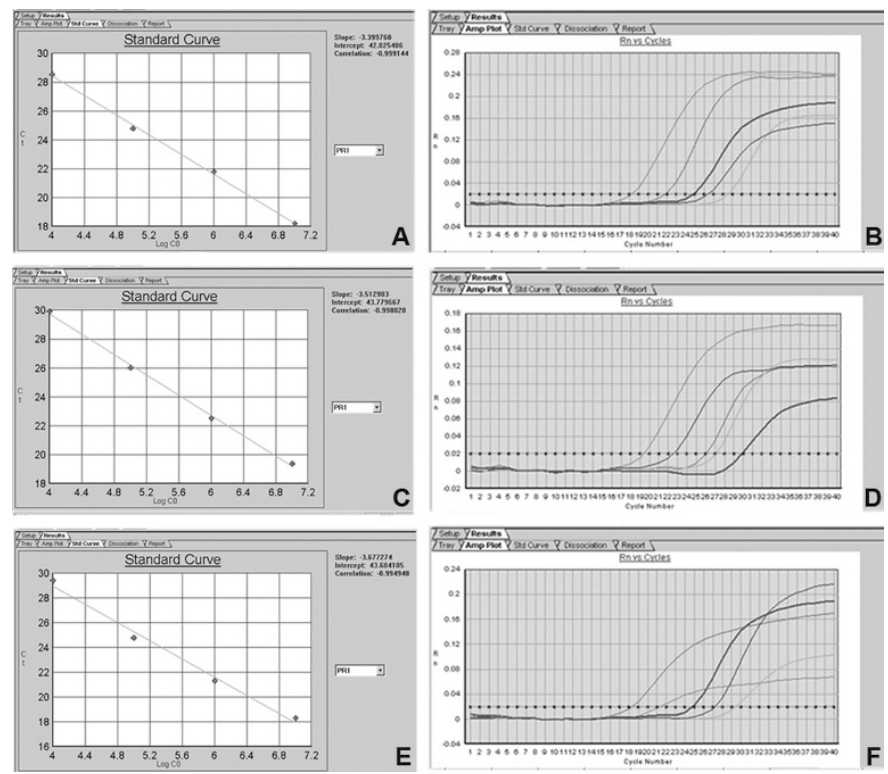

Figure 1. Real-time PCR curves. (A) Standard curves of GAPDH, (B) curves containing standard curves of GAPDH and one sample, $(C)$ standard curves of Bmall, $(D)$ curves containing standard curves of Bmall and one sample, $(E)$ standard curves of Cryl, $(F)$ curves containing standard curves of Cryl and one sample. 
Table 1. Patient characteristics

\begin{tabular}{lccc}
\hline & & No & \\
& Phototherapy & phototherapy & $p$ value \\
\hline Male/female & $20 / 12$ & $15 / 14$ & 0.395 \\
C section/vaginal & $17 / 15$ & $15 / 14$ & 0.913 \\
Total bilirubin (mg/dL) & $12.24 \pm 0.77$ & $12.06 \pm 0.76$ & 0.377 \\
Gestational age (wk) & $38.84 \pm 1.13$ & $38.59 \pm 1.05$ & 0.364 \\
Admission age $(\mathrm{d})$ & $19.43 \pm 4.28$ & $19.41 \pm 3.67$ & 0.982 \\
Weight $(\mathrm{g})$ & $3323.69 \pm 286.50$ & $3282.24 \pm 353.45$ & 0.615 \\
Core temperature $\left({ }^{\circ} \mathrm{C}\right)$ & & & \\
$\quad$ Baseline & $36.63 \pm 0.38$ & $36.58 \pm 0.20$ & 0.312 \\
$\quad$ 24 h & $36.59 \pm 0.27$ & $36.58 \pm 0.20$ & 0.808 \\
\hline
\end{tabular}

cesarean section, 12 of whom were female. In group II, 15 babies were delivered by cesarean section, 14 of whom were female. There were no differences in gender and route of delivery between the two groups $(p=0.395$ and 0.913, respectively). There were no statistical differences in terms of gestational age, postnatal age, body weight, and total serum bilirubin level before study between groups I and II $(p>0.05$ respectively). Core temperature before or after treatment in both groups was kept in the normal range.

Circadian gene mRNA abundance in PBMC. Circadian gene mRNA values were normalized to the amount of GAPDH mRNA in each sample. In group I, Bmall expression decreased after phototherapy compared with the baseline $(p=0.004)$ and Cryl expression increased following 24 hours phototherapy ( $p$ $=0.009)$. In group II, there were no significant differences in circadian gene expression between baseline and after the eyes being covered for 24 hours (Bmall: $p=0.837$, Cryl: $p=$ 0.567). There was no significant difference in baseline Bmall or Cryl expression between groups I and II ( $p>0.05$ for both) (Fig. 2). There were significant differences in the change score of circadian gene expression from baseline to postphototherapy versus baseline to post-24 hour control (Bmall: $p$ $=0.025$, Cryl: $p=0.036$ ).

Concentration of melatonin in blood. In group I, melatonin decreased following 24-hour phototherapy $(p=0.020)$. In group II, there was no significant difference in melatonin level before and 24 hours after eye covering $(p=0.927)$. There was no difference in baseline melatonin levels between groups I and II $(p>0.05)$ (Fig. 3). There was significant difference in the change of melatonin level from baseline to post-phototherapy versus baseline to post-24-hour control $(p=0.042)$.

\section{DISCUSSION}

Hyperbilirubinemia is a common problem in newborns of Chinese ethnicity, and blue light phototherapy is used as the main treatment. This therapy reduces the serum bilirubin level very effectively. No serious side effects are observed immediately upon use of phototherapy, although several well-known minor effects: cutaneous erythemas, loose green stools, changes in feeding habits, or water loss have occurred. In our clinical practice, jaundiced neonates frequently cry more and are more jittery when they were placed under the phototherapy lights. During phototherapy, the infant's eyes are routinely covered to avoid retinal damage. Consequently, the behavioral effects observed during phototherapy may be related to non-
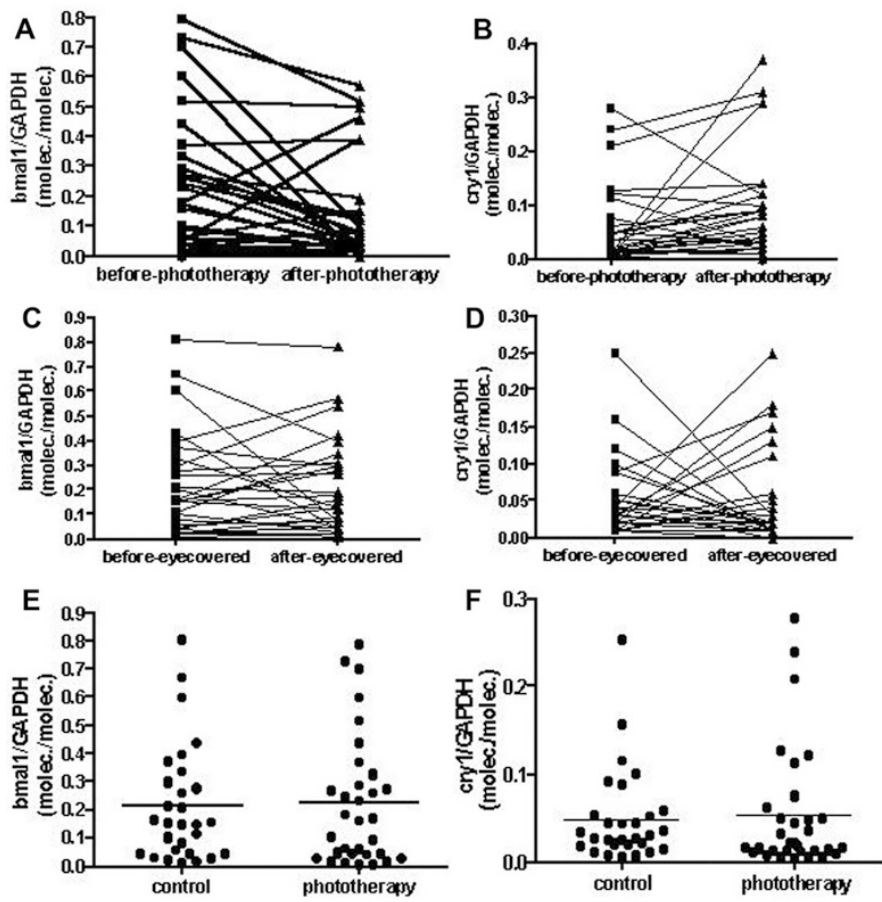

Figure 2. Circadian genes mRNA abundance normalized to GAPDH mRNA abundance in PBMC of breast-milk jaundiced neonates. In group I $(n=32)$, Bmall was lower after phototherapy than before $(p=0.004)(A)$, Cryl was higher after phototherapy than before $(p=0.009)(B)$. In group II $(n=29)$, there were no differences of Bmall and Cryl expression before and after eye-covered ( $p=0.837$ and 0.567 , respectively) $(C, D)$. $(E, F)$ There were no differences of baseline expressions of Bmall and Cry 1 between groups I and II ( $p=0.817$ and 0.378 , respectively).

ocular light stimulation and alteration in the circadian rhythm. Here we investigated two major circadian gene mRNA expressions in PBMC as well as plasma melatonin concentration to determine whether a subtle change of circadian rhythm would occur during such noninvasive therapy. To our knowledge, this is the first study to show the relationship between neonatal phototherapy and circadian rhythm disturbance.

In mammals, CLOCK and BMAL1 act as positive regulators. They bind to the E-box enhancer to activate the transcription of Period and Cry genes. PERIOD, CRY, TIMELESS, DECI, and $D E C 2$ inhibit the CLOCK and BMAL1 function. REV-ERB ${ }_{\alpha}$ repressed the transcription of Bmall. These genes constitute negative regulators (2). Studies have shown that the molecular clock mechanism oscillates not only in the central nervous system, but also in peripheral organs, such as liver, kidney, oral mucosa, fibroblast cells, and PBMC. In humans, there are only a few studies concerning the effect of light on the circadian gene expressions. In our study, the expression of Bmall was decreased and Cryl was increased in PBMC of neonates after phototherapy. The CRY has been considered as blue light receptors for plants and mammals $(12,13)$. It is possible that the upregulated expression of Cryl during blue light phototherapy could be the result of an altered dark-light environment or an intensive light exposure and a high-level Cryl expression together with other negative regulators in the transcriptionaltranslational feedback loops inhibited the expression of Bmall. By alterations in peripheral biologic oscillators, the physio- 

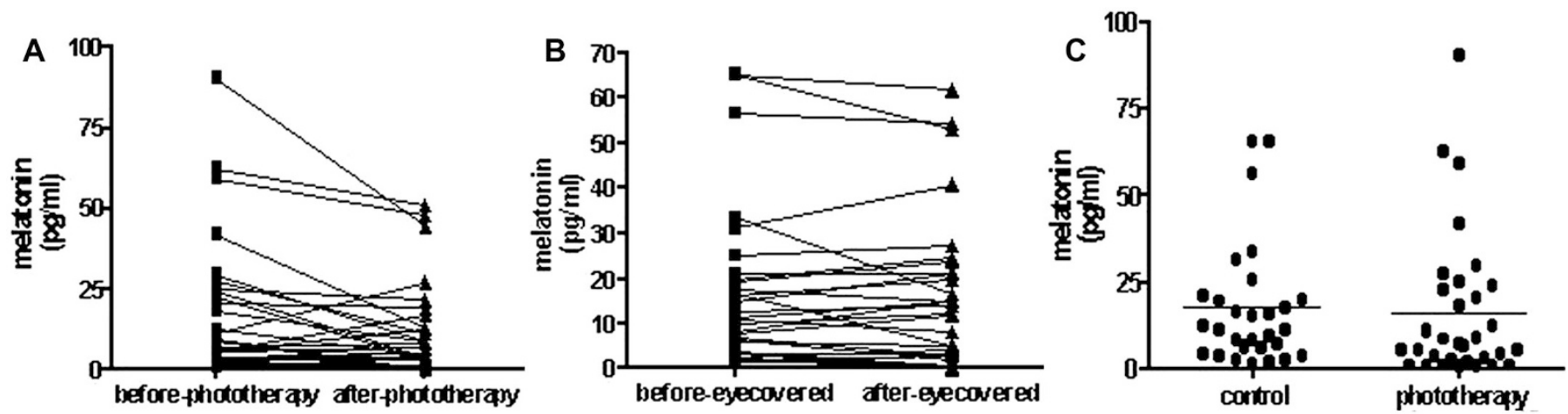

Figure 3. (A) In group I $(n=32)$, melatonin was lower after phototherapy than before $(p=0.020)$. (B) In group II $(n=29)$, there was no difference of melatonin before and after eye covering $(p=0.927)$. (C) There was no difference of baseline melatonin levels between groups I and II $(p=0.254)$.

logic and behavioral functions in neonates during phototherapy could be changed.

The relationship between the nonocular exposure to light and circadian alteration has been a controversial issue in circadian rhythm studies. Campbell and Murphy (10) documented in adult subjects that when the popliteal region was exposed to light, there were significant phase shifts in core body temperature and melatonin, whereas others insisted that dark-light stimulation to circadian system could not take place through nonocular ways $(14,15)$. In our study, the eyes both in phototherapy and controls were covered with black cloth. So the light effects on circadian rhythms by way of nonocular exposure could be observed. When two groups were compared in the study, the only treatment difference was the light intensity, strong (more than $5500 \mathrm{lux}, 806 \mu \mathrm{W} / \mathrm{cm}^{2}$ ) and unclothed exposure in phototherapy versus $\operatorname{dim}(<85$ lux $)$ and clothed in control. Significant changes in circadian gene expression in the treatment group were found when it was compared with its baseline. It is possible that prolonged, continuous, noncycling body light exposure such as a strong, 24-hour intensive phototherapy can cause changes in circadian gene expression. Our results further support the notion that nonocular light exposure can alter the expression of circadian genes in the periphery of human beings. Whether such subtle changes in circadian gene expression may translate into neonatal behavior disturbances, e.g. increased crying, jitteriness, alterations in normal heart rate or blood pressure circadian pattern, needs further study.

Melatonin is the main hormone of the pineal gland, which connects to the SCN by multisynaptic pathway. It has central analgesic actions (16) and is also considered to be a free radical scavenger (17). Low melatonin levels in infants were shown to be linked to prematurity, blindness, myoclonus, epilepsy, abnormal sleep, colic, sudden infant death syndrome, and delayed psychomotor achievements $(18,19)$. Transcript levels of some circadian clock genes were found to correlate positively and significantly with the secretion of plasma melatonin. Light exposure inhibits the secretion of melatonin and dark promotes it. The daily rhythm of melatonin appears at the end of the neonatal period and persists thereafter (20). The melatonin circadian rhythm had not yet developed in the subjects we studied. When neonates had their eyes covered, they were in the dark, and this may promote the secretion of melatonin. Conversely, in a bright and noisy nursery, the secretion of melatonin may be inhibited. After 24 hours of intensive phototherapy, the secretion of melatonin might be inhibited. In the control group, infants were clothed and the eyes were covered in a dimly lighted but noisy nursery. The promoting factors and the inhibiting factors might be balanced and melatonin levels in plasma unchanged. The results indicate that peripheral light exposure may affect the secretion of melatonin.

One of the limitations of the study is that in our neonatal nursery, the room lights are always on. Although the light intensity was considered to be $\operatorname{dim}(<85 \operatorname{lux})$ and the exposure surface was limited, it is possible that the room lights may affect the circadian rhythm and circadian gene expression. Another limitation is that we did not continuously record the babies' behaviors during the study because of the technical difficulties. We also could not observe the time lag between the peak clock gene expression and the change in melatonin concentration because of ethical concern about repeated blood sampling.

There is another limitation of our study. Although there are strong correlations between the timing and amplitude of the plasma melatonin and urinary 6-sulfatoxymelatonin rhythms (21), there is no evidence that light sources (particularly blue light frequencies) affect the half-life of melatonin. It would be useful in a further study to measure melatonin metabolic products in urine to determine whether there was increased metabolism of a fixed amount of melatonin or whether production was affected by light; it is, however, clinically difficult to obtain these urine samples.

In the recent Guideline for Perinatal Care by the American Academy of Pediatrics and American College of Obstetricians and Gynecologists, the use of a regular day-night cycle in the neonatal intensive care unit and intermediate nursery has been recommended (22). This has also been recommended by other investigators (23). In our study, the vital signs, including the body temperature, in both groups were within the normal range and no day-night effects were found. The significant alterations in circadian gene expression and melatonin secretions among neonates receiving phototherapy in our study raises questions about whether the routine phototherapy should follow a circadian pattern. The mechanism behind the altered expression of circadian genes after continuous intensive phototherapy in jaundiced neonates needs further study and investigation. 
Acknowledgments. We thank Dr. Lewis Ingram, Department of Pediatrics, Ysbyty Gwynedd, Bangor, UK; Dr. Shoo Lee, Center for Healthcare Innovation and Improvement, Canada; and Dr. William Neal, Department of Pediatrics, Health Science Center, West Virginia University for carefully reviewing the manuscript.

\section{REFERENCES}

1. Rivkees SA 2003 Developing circadian rhythmicity in infants. Pediatrics 112:373381

2. Hirota T, Fukada Y 2004 Resetting mechanism of central and peripheral circadian clocks in mammals. Zool Sci 21:359-368

3. Boivin DB, James FO, Wu A, Cho-Park PF, Xiong H, Sun ZS 2003 Circadian clock genes oscillate in human peripheral blood mononuclear cells. Blood 102:4143-4145

4. Bjarnason GA, Jordan RC, Wood PA, Li Q, Lincoln DW, Sothern RB, Hrushesky WJ, Ben-David Y 2001 Circadian expression of clock genes in human oral mucosa and skin: association with specific cell-cycle phases. Am J Pathol 158:1793-1801

5. Kennaway DJ, Wright H 2002 Melatonin and circadian rhythms. Curr Top Med Chem 2:199-209

6. Stehle JH, von Gall C, Korf HW 2003 Melatonin: a clock-output, a clock-input J Neuroendocrinol 15:383-389

7. Lowden A, Akerstedt T, Wibom R 2004 Suppression of sleepiness and melatonin by bright light exposure during breaks in night work. J Sleep Res 13:37-43

8. Lindblom N, Hatonen T, Laakso M, Alila Johansson A, Laipio M, Turpeinen U 2000 Bright light exposure of a large skin area does not affect melatonin or bilirubin levels in humans. Biol Psychiatry 48:1098-1104

9. Campbell SS, Murphy PJ, Suhner AG 2001 Extraocular phototransduction and circadian timing systems in vertebrates. Chronobiol Int 18:137-172

10. Campbell SS, Murphy PJ 1998 Extraocular circadian phototransduction in humans. Science 279:396-399
11. Zhonghua yixue hui erkexue fenghui xinshenger xuezu 2001 Intervention criteria for neonatal hyperbilirubinemia. Zhonghua Er Ke Za Zhi 39:185-187.

12. Kobayashi K, Kanno S, Smit B, van der Horst GT, Takao M, Yasui A 1998 Characterization of photolyase/blue-light receptor homologs in mouse and human cells. Nucleic Acids Res 26:5086-5092

13. Emery P, So WV, Kaneko M, Hall JC, Rosbash M 1998 CRY, a Drosophila clock and light-regulated cryptochrome, is a major contributor to circadian rhythm resetting and photosensitivity. Cell 95:669-679

14. Ruger M, Gordijn MC, Beersma DG, de Vries B, Daan S 2003 Acute and phaseshifting effects of ocular and extraocular light in human circadian physiology. J Bio Rhythms 18:409-419

15. Wright KP Jr., Czeisler CA 2002 Absence of circadian phase resetting in response to bright light behind the knees. Science 297:571-

16. Ray M, Mediratta PK, Mahajan P, Sharma KK 2004 Evaluation of the role of melatonin in formalin-induced pain response in mice. Indian J Med Sci 58:122-130

17. Gitto E, Karbownik M, Reiter RJ, Tan DX, Cuzzocrea S, Chiurazzi P, Cordaro S, Corona G, Trimarchi G, Barberi I 2001 Effects of melatonin treatment in septic newborns. Pediatr Res 50:756-760

18. Sivan Y, Laudon M, Tauman R, Zisapel N 2001 Melatonin production in healthy infants: evidence for seasonal variations. Pediatr Res 49:63-68

19. Sivan Y, Laudon M, Kuint J, Zisapel N 2000 Low melatonin production in infants with a life-threatening event. Dev Med Child Neurol 42:487-491

20. Ardura J, Gutierrez R, Andres J, Agapito T 2003 Emergence and evolution of the circadian rhythm of melatonin in children. Horm Res 59:66-72

21. Matthews CD, Guerin MV, Wang X 1991 Human plasma melatonin and urinary 6-sulphatoxy melatonin: studies in natural annual photoperiod and in extended darkness. Clin Endocrinol (Oxf) 35:21-27

22. American Academy of Pediatrics; The American College of Obstetricians and Gynecologists; American Academy of Pediatrics. Committee on Fetus and Newborn; American College of Obstetricians and Gynecologists. Committee on Obstetric Practice; March of Dimes Birth Defects Foundation 1997 Guidelines for Perinatal Care, 4th ed., Elk Grove Village, and Washington, DC, pp 47-48.

23. Ariagno RL, Mirmiran M 2001 Shedding light on the very low birth weight infant J Pediatr 139:476-477 